\title{
Análisis del uso de antibióticos en antibiogramas de urocultivos realizados por un laboratorio clínico de la región centro-occidental de Colombia
}

Analysis of the use of antibiotics in antibiograms of urine carried out by a clinical laboratory of the central-western region of Colombia

\author{
Carlos Rodríguez-Salazar ${ }^{1,2^{*}}$ orcid.org/0000-0002-0071-1289 \\ Delia Recalde-Reyes ${ }^{1,2}$ orcid.org/0000-0002-7752-1221 \\ Leonardo Padilla-Sanabria1,2 orcid.org/0000-0001-5811-2791
}

1 Grupo de Investigación en Gestión del Conocimiento en Salud, Corporación Universitaria Empresarial Alexander von Humboldt. Armenia, Colombia

2 Grupo de Inmunología Molecular (GYMOL), Universidad del Quindío. Armenia, Colombia

Fecha de recepción: Noviembre 4-2016 Fecha de revisión: Agosto 24-2017 Fecha de aceptación: Diciembre 1 - 2017

Rodríguez-Salazar C, Recalde-Reyes D, Padilla-Sanabria L. Análisis del uso de antibióticos en antibiogramas de urocultivos realizados por un laboratorio clínico de la región centro-occidental de Colombia. Univ. Salud. 2017;19(3):378-387. D0I: http://dx.doi.org/10.22267/rus.171903.100

\section{Resumen}

Introducción: Los antibióticos son moléculas bactericidas/bacteriostáticas que controlan infecciones bacterianas, su uso incorrecto favorece multirresistencia o falla terapéutica en el caso de cepas bacterianas naturalmente resistentes, generando así un riesgo para la salud. Objetivo: Analizar el uso de antibióticos en antibiogramas de urocultivos realizados por un laboratorio clínico (región centro-occidental, Colombia). Materiales y métodos: Estudio descriptivo-retrospectivo. Se tomaron datos de urocultivos y antibiogramas realizados entre abril de 2014 a junio de 2015 por un laboratorio clínico de la región centro-occidental de Colombia. Los datos obtenidos fueron confrontados con los protocolos descritos por el Instituto Nacional de Salud de Colombia. Resultados: Se analizaron 1815 reportes de urocultivos y antibiogramas, identificando 18 especies bacterianas. En el 22,3\%(403) de casos se evaluaron y reportaron antibióticos sobre microorganismos naturalmente resistentes. Pseudomonas aeruginosa presentó mayor resistencia, el antibiótico con mayor resistencia fue ácido nalidíxico (66,7\%). Conclusión: El estudio mostró que existe un problema en cuanto al manejo, reporte e interpretación de antibiogramas frente a microorganismos naturalmente resistentes, que podría favorecer el desarrollo de multirresistencia en microorganismos sensibles de la flora bacteriana. Una revisión de la bibliografía nacional e internacional, mostró reportes similares; ningún autor menciona resistencias intrínsecas, por lo que los datos de antibiótico resistencia serían sobreevaluados.

Palabras clave: Antibióticos; antibiograma; farmacorresistencia bacteriana múltiple. (Fuente: DeCS, Bireme).

\begin{abstract}
Introduction: Antibiotics are bactericidal/bacteriostatic molecules that control bacterial infections, its misuse favors multidrug or therapeutic failure in the case of naturally resistant bacterial strains, thus generating a health risk. Objective: To analyze the use of antibiotics in urine antibiograms carried out by a clinical laboratory (centralwestern region, Colombia). Materials and methods: A descriptive-retrospective study was made. Urine and antibiograms data were collected from April 2014 to June 2015 by a clinical laboratory in the central-western region of Colombia. The obtained data was confronted with the protocols described by the National Institute of Health of Colombia. Results: 1815 reports of urine and antibiograms were analyzed, identifying 18 bacterial species. In the $22.3 \%$ (403) of cases, antibiotics were evaluated and reported on naturally resistant microorganisms. Pseudomonas
\end{abstract}


aeruginosa showed greater resistance and the antibiotic with the highest resistance was nalidixic acid (66.7\%). Conclusion: The study showed that there is a problem in managing, reporting and interpreting antibiograms against naturally resistant microorganisms, which could favor the development of multidrug in sensitive microorganisms of bacterial flora. A review of national and international bibliography showed similar reports; however, no author mentions intrinsic resistances, so the data of antibiotic resistance would be over evaluated.

Keywords: Antibiotics; microbial sensitivity test; drug resistance, multiple, bacterial. (Source: DeCS, Bireme).

\section{Introducción}

La resistencia bacteriana es un problema de salud pública a nivel mundial. El uso indiscriminado de los antibióticos ha favorecido la presión selectiva en los microorganismos y en consecuencia la efectividad de tratamientos antimicrobianos ha disminuido. Este tipo de falla terapéutica ha generado en primer lugar: altos costos en el sistema de salud (mayores tiempos de hospitalización y cambios de diversos esquemas antimicrobianos) y en segundo lugar, pero no menos importante: incremento en las tasas de morbimortalidad(1-3).

Desde el campo del laboratorio clínico, los conceptos establecidos en cuanto a la interpretación de pruebas de susceptibilidad dadas por el Instituto de Normas Clínicas y de Laboratorio (CLSI por sus siglas en inglés) o por el Comité Europeo de Pruebas de Susceptibilidad a los Antimicrobianos (EUCAST por sus siglas en ingles), son modificados constantemente y tanto en el ámbito médico como del laboratorio, se desconocen o se pasan por alto estas normas de interpretación(4-7).

En algunos casos tanto los clínicos como laboratoristas desconocen, los procesos de resistencia intrínseca y extrínseca, propias de los microorganismos (porinas, bombas e-flujo, perdida de permeabilidad, enzimas codificadas genéticamente o por plásmidos, sobreproducción, protección, mutación del blanco molecular o bypass metabólico), los cuales son fundamentales para una adecuada interpretación de los antibiogramas y como consecuencia, un manejo terapéutico oportuno y eficaz $^{(7-9)}$. Por lo anterior, el presente estudio tuvo por objetivo analizar el uso correcto de antibióticos en antibiogramas provenientes de urocultivos realizados por un laboratorio clínico de la región centro-occidental de Colombia.

\section{Materiales y métodos}

Se realizó un estudio descriptivo retrospectivo entre abril de 2014 a junio de 2015 donde se tomaron datos de urocultivos y antibiogramas que fueron realizados por un laboratorio clínico de mediana complejidad de la región centrooccidental de Colombia, el cual atiende diariamente en promedio 250 pacientes ambulatorios de diferentes empresas promotoras de salud (EPS), con una mayor frecuencia de adultos mayores. De acuerdo a los datos suministrados por el laboratorio, todas las muestras fueron recolectadas por micción espontánea. El análisis de estos resultados incluyó: microorganismo aislado e identificación (el cual fue realizado en medio cromogénico CPS)(10), susceptibilidad o resistencia a los antibióticos (método de Kirby \& Bauer) y datos demográficos; los cuales se sistematizaron en una hoja de cálculo de Excel. Se realizó estadística descriptiva mediante el paquete estadístico Graphpad Prism v 6,04 (San Diego California, USA). La comparación de la prevalencia de los uropatógenos con el grupo etario se realizó a través la prueba Chi Cuadrado de Pearson, con un nivel de confiabilidad del 0,05; los datos obtenidos fueron confrontados con los protocolos descritos en el manual de procedimientos para la determinación de susceptibilidad antibiótica en patógenos de importancia hospitalaria del Instituto Nacional de Salud (INS) el cual, se basa en las normas y recomendaciones del Instituto de Estándares de Laboratorios Clínicos (CLSI) ${ }^{(11,12) .}$

\section{Consideraciones éticas}

Dentro del marco legal que ampara este proyecto se encuentra la Resolución 8430 de 1993 del Ministerio de Salud y Protección Social, que abarca las normativas que deben aplicarse en procesos de investigación científica. Dado que el 
proyecto no involucra ensayos con organismos vivos se clasifica como un trabajo de riesgo mínimo, adicionalmente los datos obtenidos fueron manejados con total discreción bajo anonimato y en ningún momento se revelan datos que afecten la integridad humana, el manejo de los microorganismos se realizó bajo control de la institución, siguiendo todas las prácticas de bioseguridad. El trabajo contó con el aval del laboratorio clínico para la revisión de historias clínicas y fue aprobado por el Comité de Bioética de la Corporación Universitaria Empresarial Alexander von Humboldt de la ciudad de Armenia y debido a que no se practicaron procedimientos experimentales con humanos, no se solicitó un aval para dichos procesos.

\section{Resultados}

Para el periodo de estudio se realizaron un total de 1815 aislados con su respectivo antibiograma. De estos un $85 \%$ pertenecieron a pacientes del género femenino (Tabla 1) predominando los adultos mayores con un 45\% (Tabla 2).

Tabla 1. Descripción de la población de estudio

\begin{tabular}{llrrrr}
\hline \multirow{2}{*}{ Características } & \multicolumn{2}{c}{$\mathbf{N}^{\circ}$} & \multicolumn{3}{c}{ IC 95\% } \\
& casos & Límite & $\begin{array}{c}\text { Límite } \\
\text { inferior } \\
\text { mayor }\end{array}$ \\
\hline Año & & & & & \\
& 2014 & 104 & 44,3 & 42 & 46,6 \\
& 2015 & 55,7 & 53,4 & 58 \\
Sexo & & & & \\
& Femenino & 1559 & 85,9 & 84,2 & 87,5 \\
& Masculino & 240 & 13,2 & 11,7 & 14,9 \\
& Sin determinar & 16 & 0,9 & 0,5 & 1,4 \\
Grupo etario & & & & \\
& Niño & 180 & 9,9 & 8,6 & 11,4 \\
& Adolescente & 75 & 4,1 & 3,3 & 5,2 \\
& Adulto joven & 321 & 17,7 & 16 & 19,5 \\
& Adulto medio & 417 & 22,9 & 21,1 & 25 \\
& Adulto mayor & 818 & 45,1 & 42,8 & 47,4 \\
& Sin edad & 4 & 0,2 & 0,1 & 0,6 \\
\hline
\end{tabular}

(La edad se categorizó en: i) niños entre 0 y 10 años, ii) adolescentes entre 11 y 20 , iii) adultos jóvenes entre 21 y 44 , iv) adultos medios 45 y 64, y v) adultos mayores a partir de los 65 años.) Se utilizó un Intervalo de confianza del 95\% (IC: 95\%).

Tabla 2. Comparación de las prevalencias de las infecciones con cada microorganismo según grupo etario

\begin{tabular}{|c|c|c|c|c|c|c|c|}
\hline Prevalencia \% (\#) & Niño & Adolescente & $\begin{array}{c}\text { Adulto } \\
\text { joven }\end{array}$ & $\begin{array}{c}\text { Adulto } \\
\text { medio }\end{array}$ & Adulto mayor & Sin edad & Valor $\mathbf{p} \mathbf{C h}^{2}$ \\
\hline Escherichia coli. & $41(74)$ & $42,7(32)$ & $48,9(157)$ & $66,9(279)$ & $60,4(494)$ & $100(4)$ & 0,0001 \\
\hline Enterococcus faecalis & $21,6(39)$ & $28(21)$ & $26,2(84)$ & $9,8(41)$ & $11,1(91)$ & 0 & 0,0001 \\
\hline Enterobacter cloacae & $15(27)$ & $21,3(16)$ & $15,3(49)$ & $12,7(53)$ & $14,9(122)$ & 0 & 0,44 \\
\hline Citrobacter spp & $5,5(10)$ & 0 & $2,2(7)$ & 4,3 (18) & $4,9(40)$ & 0 & 0,12 \\
\hline Proteus spp & $10,5(19)$ & $1,3(1)$ & $2,5(8)$ & $2,9(12)$ & $3,2(26)$ & 0 & 0,0001 \\
\hline Pseudomonas aeruginosa & $2,7(5)$ & $5,3(4)$ & 0 & $0,6(3)$ & $2,1(17)$ & 0 & 0,0049 \\
\hline Klebsiella spp & $1,6(3)$ & 0 & $2,8(9)$ & $1,2(5)$ & $2,3(19)$ & 0 & 0,46 \\
\hline Staphylococcus spp & $1,1(2)$ & $1,3(1)$ & $1,9(6)$ & $1,4(6)$ & $1(8)$ & 0 & 0,89 \\
\hline Serratia spp & $0,5(1)$ & 0 & 0 & 0 & $0,1(1)$ & 0 & 0,5272 \\
\hline Morganella spp & $0,5(1)$ & 0 & 0 & $0,2(1)$ & 0 & 0 & 0,3892 \\
\hline
\end{tabular}

Escherichia coli fue la enterobacteria más prevalente, en las muestras analizadas con un porcentaje global de $57,3 \%$ (Tabla 3 ).

En el 22,3\% (403) de los casos se evaluaron y reportaron antibióticos sobre microorganismos naturalmente resistentes, como en el caso de algunos aislados de Pseudomonas aeruginosa, Proteus spp, Enterococcus faecalis, Enterobacter cloacae spp. y Klebsiella pneumoniae (Tabla 4).
Tabla 3. Prevalencia de uropatógenos aislados en las muestras analizadas

\begin{tabular}{lrrr}
\hline \multirow{2}{*}{ Uropatógenos } & $\begin{array}{c}\text { Porcentaje } \\
\text { global \% }\end{array}$ & $\begin{array}{c}\text { Límite } \\
\text { inferior }\end{array}$ & $\begin{array}{l}\text { IC 95\% } \\
\text { Lámite } \\
\text { mayor }\end{array}$ \\
\hline Escherichia coli & 57,3 & 54,9 & 59,6 \\
Enterococcus spp & 15,2 & 13,6 & 16,9 \\
Enterobacter spp & 14,7 & 13,1 & 16,4 \\
Citrobacter spp & 4,1 & 3,3 & 5,2 \\
Proteus spp & 3,6 & 2,8 & 4,6 \\
Pseudomonas spp & 1,6 & 1,1 & 2,3 \\
Klebsiella spp & 2 & 1,4 & 2,7 \\
Staphylococcus spp & 1,3 & 0,8 & 1,9 \\
Serratia spp & 0,1 & 0,01 & 0,4 \\
Morganella spp & 0,1 & 0,001 & 0,3 \\
\hline Índice de Confiabilidad (IC: 95\%) para una proporción
\end{tabular}


Tabla 4. Perfil de Sensibilidad, Sensibilidad Intermedia y Resistencia de los microorganismos a diferentes antibióticos

\begin{tabular}{|c|c|c|c|c|c|c|c|c|}
\hline Prevalencia \% (\#) & E. coli & E. faecalis & P. spp. & P. aeruginosa & E. cloacae & C. spp. & K. spp & S. spp \\
\hline \multicolumn{9}{|l|}{ Ácido nalidíxico } \\
\hline $\mathrm{s}$ & $28,2(231)$ & $25(10)$ & $53,7(29)$ & $9,09(1)$ & $36,9(83)$ & $39,3(24)$ & $31,3(10)$ & $12,5(1)$ \\
\hline & $1,46(12)$ & 0 & $5,56(3)$ & 0 & $2,22(5)$ & $4,92(3)$ & $9,38(3)$ & 0 \\
\hline & $70,4(577)$ & $75(30)$ & $40,7(22)$ & $90,9(10)$ & $60,9(137)$ & $55,7(34)$ & $59,4(19)$ & $87,5(7)^{*}$ \\
\hline \multicolumn{9}{|l|}{ Amikacina } \\
\hline $\mathrm{s}$ & $90,2(413)$ & $75(15)$ & $92,6(25)$ & $87,5(14)$ & $81,7(89)$ & $91,7(33)$ & $95(19)$ & $75(3)$ \\
\hline & $1,09(5)$ & 0 & 0 & 0 & 0 & 0 & 0 & 0 \\
\hline & $8,73(40)$ & $25(5)^{* *}$ & $7,41(2)$ & $12,5(2)$ & $18,3(20)$ & $8,33(3)$ & $5(1)$ & $25(1)$ \\
\hline \multicolumn{9}{|l|}{ Ampicilina } \\
\hline $\mathrm{S}$ & $29,8(121)$ & $95(248)$ & $53,6(15)$ & 0 & $21,6(25)$ & $44,4(16)$ & $13,3(2)$ & $64,3(9)$ \\
\hline & 2,71 (11) & $0,38(1)$ & 0 & 0 & 0 & 2,78 (1) & $20(3)$ & $14,3(2)$ \\
\hline & $67,5(274)$ & $4,6(12)$ & $46.4(13)^{*}$ & $100(3)^{*}$ & $78,4(91)^{*}$ & $52,8(19)^{*}$ & $66,7(10)$ & $21,4(3)$ \\
\hline \multicolumn{9}{|l|}{ Ampicilina/sulbactam } \\
\hline $\mathrm{S}$ & 77 (385) & $88,2(15)$ & $80(40)$ & $18,2(2)$ & $56,6(43)$ & $72,2(13)$ & $73,9(17)$ & $85,7(12)$ \\
\hline & $6,8(34)$ & 0 & $6(3)$ & 0 & $9,21(7$ & 0 & $8,7(2)$ & 0 \\
\hline & $16,2(81)$ & $11,8(2)$ & $14(7)$ & $81,8(9)^{*}$ & $34,2(26)^{*}$ & $27,8(5)$ & $17,4(4)$ & $14,3(2)$ \\
\hline \multicolumn{9}{|l|}{ Cefalotina } \\
\hline $\mathrm{S}$ & $38,4(211)$ & $76,7(23)$ & $56(14)$ & $20(2)$ & $31,7(52)$ & $41,5(17)$ & $70(7)$ & $75(3)$ \\
\hline & $12,6(69)$ & 3,33(1) & $12(3)$ & 0 & $4,27(7)$ & $4,88(2)$ & $10(1)$ & 0 \\
\hline & 49 (269) & $20(6)^{* *}$ & $32(8)^{*}$ & $80(8)$ & $64(105)^{*}$ & $53,7(22)$ & $20(2)$ & $25(1)$ \\
\hline \multicolumn{9}{|l|}{ Cefoxitina } \\
\hline $\mathrm{S}$ & $90(180)$ & 85,7 (6) & 71,4 (15) & $9,09(1)$ & $53,8(28)$ & $60(6)$ & $63,6(7)$ & $100(5)$ \\
\hline & $3,5(7)$ & 0 & $19(4)$ & 0 & $7,69(4)$ & $20(2)$ & 9,09 (1) & 0 \\
\hline $\mathrm{R}$ & 6,5 (13) & $14,3(1)^{* *}$ & $9,52(2)$ & $90,9(10)$ & $38,5(20)^{*}$ & $20(2)$ & $27,3(3)$ & 0 \\
\hline \multicolumn{9}{|l|}{ Ceftazidima } \\
\hline $\mathrm{S}$ & 88,3 (273) & 75 (3) & $81,8(18)$ & $88,9(16)$ & $71,1(32)$ & 72,7 (16) & $92,3(12)$ & $100(4)$ \\
\hline & $1,94(6)$ & 0 & 4,55 (1) & 0 & $4,44(2)$ & 0 & 0 & 0 \\
\hline & $9,71(30)$ & $25(1)^{* *}$ & $13,6(3)$ & $11,1(2)$ & 24,4 (11) & $27,3(6)$ & 7,69 (1) & 0 \\
\hline \multicolumn{9}{|l|}{ Ceftriaxona } \\
\hline S & 80,2 (239) & $60(3)$ & $87(20)$ & $37,5(6)$ & $60,6(20)$ & $58,8(10)$ & $80(12)$ & $100(1)$ \\
\hline & $4,36(13)$ & 0 & 0 & $25(4)$ & $3,03(1)$ & $5,88(1)$ & 0 & 0 \\
\hline $\mathrm{R}$ & $15,4(46)$ & $40(2)^{* *}$ & $13(3)$ & $37,5(6)^{*}$ & $36,4(12)$ & $35,3(6)$ & $20(3)$ & 0 \\
\hline \multicolumn{9}{|l|}{ Ciprofloxacina } \\
\hline $\mathrm{S}$ & $45,4(453)$ & 68,8 (33) & $90,3(56)$ & $69,6(16)$ & 60,1 (155) & $64,4(47)$ & $55,9(19)$ & $50(8)$ \\
\hline & $0,8(8)$ & $2,08(1)$ & $3,23(2)$ & 0 & $1,94(5)$ & 0 & $2,94(1)$ & 0 \\
\hline $\mathrm{R}$ & $53,8(536)$ & $29,2(14)$ & $6,45(4)$ & 30,4 (7) & $38(98)$ & $35,6(26)$ & $41,2(14)$ & $50(8)$ \\
\hline \multicolumn{9}{|l|}{ Gentamicina } \\
\hline $\mathrm{S}$ & $76(276)$ & 83,3 (10) & 88,9 (32) & $100(3)$ & $76,9(30)$ & 76,5 (13) & $93,8(15)$ & $77,8(7)$ \\
\hline & $0,55(2)$ & 0 & 0 & 0 & $2,56(1)$ & 0 & 0 & 0 \\
\hline $\mathrm{R}$ & 23,4 (85) & $16,7(2)^{* *}$ & $11,1(4)$ & 0 & $20,5(8)$ & $23,5(4)$ & 6,25 (1) & $22,2(2)$ \\
\hline \multicolumn{9}{|l|}{ Nitrofurantoína } \\
\hline $\mathrm{S}$ & $83,9(840)$ & $88,5(46)$ & $8,77(5)$ & $9,09(1)$ & $56,7(144)$ & $37,1(26)$ & $66,7(22)$ & $62,5(5)$ \\
\hline & $2,6(26)$ & $1,92(1)$ & $1,75(1)$ & 0 & $4,72(12)$ & $1,43(1)$ & $12,1(4)$ & $25(2)$ \\
\hline & 13,5 (135) & $9,62(5)$ & $89,5(51)^{*}$ & $90,9(10)$ & $38,6(98)$ & $61,4(43)$ & $21,2(7)$ & 12,5 (1) \\
\hline \multicolumn{9}{|c|}{ Trimetoprima/sulfametoxazol } \\
\hline $\mathrm{s}$ & $45,4(459)$ & $68(34)$ & $55,6(35)$ & $7,69(1)$ & $49,6(127)$ & $49,3(36)$ & $44,4(16)$ & $21,1(4)$ \\
\hline & $0,49(5)$ & 0 & $1,59(1)$ & 0 & $0,78(2)$ & 0 & 0 & $5,26(1)$ \\
\hline $\mathrm{R}$ & $54,1(547)$ & $32(16)^{* *}$ & $42,9(27)$ & $92,3(12)^{*}$ & $49,6(127)$ & $50,7(37)$ & $55,6(20)$ & $73,7(14)$ \\
\hline \multicolumn{9}{|l|}{ Imipenem } \\
\hline $\mathrm{s}$ & $93,4(99)$ & 0 & 0 & $100(17)$ & $93,1(27)$ & $87,5(7)$ & 0 & 0 \\
\hline & 0 & 0 & 0 & 0 & 0 & 0 & 0 & 0 \\
\hline & $6,6(7)$ & 0 & 0 & 0 & $6,9(2)$ & $12,5(1)$ & 0 & 0 \\
\hline \multicolumn{9}{|l|}{ Piperacilina-Tazobactam } \\
\hline $\mathrm{s}$ & $89,5(102)$ & 0 & 0 & $87,5(14)$ & $86,7(26)$ & $100(8)$ & 0 & 0 \\
\hline & $5,26(6)$ & 0 & 0 & 0 & 0 & 0 & 0 & 0 \\
\hline $\mathrm{R}$ & $5,26(6)$ & 0 & 0 & $12,5(2)$ & $13,3(4)$ & 0 & 0 & 0 \\
\hline
\end{tabular}

Omitiendo los datos en los que se emplearon antibióticos frente a microrganismos naturalmente resistentes, se encontraron los siguientes porcentajes de resistencia por los microorganismos evaluados (Tabla 5).
Omitiendo las resistencias intrínsecas por parte de los microorganismos a algunos antibióticos, se obtuvo el porcentaje de antibióticos que mostraron mayor resistencia en los antibiogramas realizados (Tabla 6). 
Tabla 5. Porcentajes de microorganismos resistentes a los diferentes antibióticos

\begin{tabular}{lr}
\hline \multicolumn{1}{c}{ Microorganismo } & \% resistencia a antibióticos \\
\hline Pseudomonas aeruginosa & 17,3 \\
Citrobacter spp & 17 \\
E. coli & 16,5 \\
Klebsiella spp. & 14,3 \\
Enterobacter cloacae & 12,6 \\
Staphylococcus spp. & 10 \\
Proteus spp. & 6,5 \\
Enterococcus faecalis & 5,3 \\
\hline
\end{tabular}

Tabla 6. Porcentaje de resistencia que mostraron los microorganismos evaluados a cada uno de los antibióticos

\begin{tabular}{lr}
\hline \multicolumn{1}{c}{ Antibiótico } & $\begin{array}{r}\text { \% de resistencia } \\
\text { microorganismos resistentes) }\end{array}$ \\
\hline Ácido nalidíxico & $66,6(829)$ \\
Trimetoprima/sulfametoxazol & $51,7(772)$ \\
Ciprofloxacina & $46,8(707)$ \\
Cefalotina & $42,3(302)$ \\
Ampicilina & $39,7(299)$ \\
Gentamicina & $21,1(104)$ \\
Nitrofurantoína & $20,3(299)$ \\
Ceftriaxona & $17,5(70)$ \\
Ampicilina/sulbactam & $14,9(101)$ \\
Ceftazidima & $12,2(53)$ \\
Cefoxitina & $10,1(30)$ \\
Amikacina & $10,1(69)$ \\
Piperacilina-Tazobactam & $7,1(12)$ \\
Imipenem & $6,3(10)$ \\
\hline
\end{tabular}

\section{Discusión}

La antibiótico-resistencia es un problema de salud pública con altas tasas de incidencia, prevalencia y morbi-mortalidad a nivel mundial, por lo ello muchos de los esfuerzos están dirigidos a su control. Pese a ello, estas iniciativas se ven obstaculizadas por factores fundamentales como la dificultad de acceso a las moléculas antimicrobianas adecuadas o la falta de formación por parte del personal de salud, debido a que en muchas ocasiones la poca fundamentación teórica en el momento de toma de decisiones terapéuticas, trae como consecuencia un incremento en la incidencia y prevalencia de infecciones con bacterias multirresistentes ${ }^{(13,14)}$.
Los resultados de este trabajo mostraron que el género femenino fue más susceptible a infecciones del tracto urinario $(85,9 \%)$ respecto a los hombres $(13,2 \%)$, también se observó que estas infecciones fueron más frecuentes en adultos mayores, resultados similares fueron reportados por Orrego et al.(15) y Barranco et al.(16), quienes reportaron que el 74,8\% y 72,9\% de las mujeres presentaron infección urinaria, seguido por un $25,2 \%$ y $27,1 \%$ de los hombres; esto se ha relacionado con el uso de ropa ajustada, relaciones sexuales, el uso de espermicidas $o$ de diafragmas vaginales, incontinencia, volumen de orina residual, niveles bajos de estrógeno y disminución de la micro flora vaginal, que facilitan la infección del tracto genitourinario, frecuentemente afecta más a mujeres, sin embargo, el hombre no está exento de infección y explicaría los resultados encontrados en el presente trabajo(17,18).

En este estudio se encontró que el 57,3\% de los aislados clínicos reportados correspondieron a $E$. coli, teniendo en cuenta que este microorganismo es uno de los más abundantes en la microbiota, es común encontrarlo causando problemas en el tracto urinario, con porcentajes de prevalencia entre el $60 \%$ y $85 \%$ de acuerdo con lo reportado en la literatura(19-25). El 70,4\% de los aislados de E. coli, mostró resistencia a varios antibióticos, datos preocupantes ya que esta bacteria no se le ha reportado resistencia intrínseca(11); así las opciones terapéuticas se reducen progresivamente por presencia de mecanismos de resistencia, algunos de ellos son la presencia de plásmidos que codifican betalactamasas de espectro extendido (BLEE), que les permite hidrolizar penicilinas, oximinocefalosporinas, cefalosporinas de espectro extendido y aztreonam. Adicionalmente pueden conferirle resistencia a aminoglucósidos, tetraciclinas, trimetoprima/sulfametoxazol y quinolonas. La producción de mutaciones puntuales genera cambios del sitio donde actúa el antibiótico, aumentando el porcentaje de resistencia, de igual manera, la presencia de bombas de eflujo, complican aún más el panorama, ya que estos microrganismos son resistentes a casi todos los antibióticos que existen en el mercado(26). 
El segundo microorganismo más reportado en este estudio fue E. faecalis, al igual que E. coli es un residente natural del intestino y se ha reportado como uno de los uropatógenos más frecuentes en infecciones del tracto urinario(15). El tratamiento de elección es ampicilina, pero también puede evaluarse penicilina, levofloxacina nitrofurantoína para aislados de orina fosfomicina principalmente. En el presente trabajo E. faecalis mostró resistencia a ampicilina en un 4,6\%, mientras que en trabajos de Machado et al.(27), reporta para este microrganismo resistencia a ampicilina del 53\% y Orrego y Enao(15) evidencian un porcentaje de resistencia a ampicilina del 14\%. Antibióticos como cefalosporinas, aminoglucósidos, clindamicina, trimetoprima/sulfametoxazol pueden ser activos in vitro, pero clínicamente no son efectivos, por ello, no deberían ser evaluados y menos reportados como susceptibles; aspecto importante a destacar, ya que en el presente trabajo se encontró que algunos aislados fueron reportados como sensibles a estos antibióticos y su uso no sería clínicamente efectivo(11).

Cuando se analizó el género Staphylococcus spp., se encontró que los antibióticos que deben ser analizados por antibiograma en atención primaria son: penicilina, oxacilina, gentamicina, tobramicina, moxifloxacino, eritromicina, clindamicina, cotrimoxazol, levofloxacina y nitrofurantoína siendo estos dos últimos importantes para los aislados en orina(11). Se encontró que hubo poca resistencia a la nitrofurantoína $(12,5 \%)$ por lo que podría considerarse para su manejo, cabe destacar que la nitrofurantoína, es utilizado como antiséptico urinario y en muchos casos se prescribe por varios meses a bajas dosis en pacientes convalecientes de una infección urinaria, especialmente en aquellos que presentan infecciones a repetición(19,20). Por otro lado, el trimetoprima/sulfametoxazol, ciprofloxacina, gentamicina y ampicilina, presentaron resistencias de 73,2\%; 50\%; 22,2\%; 21,4\% respectivamente. Se encontró adicionalmente reportes de resistencia del $87,5 \%$ al ácido nalidíxico, sin embargo, según el CLSI Staphylococcus spp., posee resistencia intrínseca a ese antibiótico por tal motivo no debería reportarse ni evaluarse en el antibiograma(11).

E. cloacae y Citrobacter spp., presentaron resistencia a ácido nalidíxico (60,9\% y 55,7\%), trimetoprima/sulfametoxazol $(49,6 \%$ y $50,7 \%)$ y nitrofurantoína $(38,6 \%$ y $61,4 \%)$, se ha reportado una alta expresión betalactamasa cromosómica inducible con actividad cefalosporinasa que, en general les confiere resistencia a aminopenicilinas y cefalosporinas de primera generación, razón por la cual estos microorganismos presentan alta tasas de resistencia y no debería por lo tanto evaluarse antibióticos, sin embargo se observó que hubo reportes de ampicilina en el estudio. Adicionalmente, en el presente trabajo, no se evalúo la presencia de betalactamasas debido a que fueron datos recolectados directamente de los reportes de laboratorio(28).

El análisis para aislados de Klebsiella spp., mostró resistencia para: nitrofurantoína, ácido nalidíxico, trimetoprima/sulfametoxazol, cefalotina $\quad(21,2 \%, \quad 66,7 \%, \quad 55,6 \%, \quad 20 \%$ respectivamente). Para Klebsiella spp., las posibilidades de tratamientos se reducen, teniendo en cuenta que codifica cromosómicamente la betalactamasa SHV-1 que la hace naturalmente resistente a ampicilina, además tiene la posibilidad de adquirir BLEE, lo cual genera resistencia a cefalosporinas y probablemente monobactámicos y carbapenémicos(11,28,29).

Se encontró $P$. aeruginosa en un $1,6 \%$ de los urocultivos, reportes similares se presentaron en otros trabajos en Colombia donde se reportó: 1,8\% de aislados en Medellín, 1,27\% en Soledad (Atlántico), $0,24 \%$ en Pereira(15,16,27). Los antibióticos a informar generalmente son ciprofloxacina, levofloxacina, gentamicina, tobramicina, fosfomicina; adicionalmente, para aislados urinarios debe reportase norfloxacina y ofloxacina(11). P. aeruginosa puede presentar múltiples mecanismos de resistencia tales como: alteraciones de la permeabilidad de membrana, bombas de eflujo y mutaciones de las porinas transmembranales; posee dos clases de $\beta$ lactamasas: Amp-C codificada en el cromosoma 
bacteriano e inducida por los propios $\beta$ lactámicos, especialmente cefalotina y ampicilina y las $\beta$-lactamasas de espectro extendido (BLEE), esto explicaría las resistencias encontradas, particularmente las $\operatorname{BLEE}^{(30)}$ explicarían la resistencia a las demás cefalosporinas y monobactámicos como el aztreonam. No se observó resistencia a imipenem, lo cual permite inferir que no presentaban carbapenemasas. En los antibiogramas analizados se encontró que se realizaron reportes de evaluación a ampicilina, ampicilina/sulbactam, ceftriaxona. trimetoprima/sulfametoxazol, sin tener presente que $P$. aeruginosa posee resistencia intrínseca o natural a estos, por lo que es indispensable conocer estos mecanismos y mejorar el panel de evaluación(11).

Es importante reconocer que en el presente trabajo se encontraron reportes de antibióticos evaluados sobre bacterias que poseen resistencia intrínseca, el desconocimiento en el manejo y uso de los antibióticos. Al realizar una búsqueda bibliográfica en fuentes nacionales e internacionales, se encontró que son comunes los reportes de evaluación de antibióticos sobre bacterias que naturalmente o de forma innata resisten su modo de acción, tal es el caso de Orrego y Enao et al.(15), que al igual que en el presente estudio, tomaron datos de urocultivos y antibiogramas realizados en un laboratorio clínico de Medellín (Colombia), encontrando que se había evaluado nitrofurantoína en Proteus spp., ampicilina, y ampicilina/sulbactam en $P$. aeruginosa y reportando como resistentes; estos microorganismos poseen resistencia intrínseca a dichos antibióticos y no deberían reportarse.

Por otro lado, Machado et al.(27), encontraron reportes de cefalosporinas y trimetoprima/sulfametoxazol en Enterococcus spp., teniendo en cuenta la resistencia intrínseca de estos microorganismos, esos antibióticos evaluados pueden ser activos in vitro, pero clínicamente no son efectivos y no deberían ser reportados como susceptibles. En el reporte de Vélez et al.(31), de datos de urocultivos obtenidos de un centro hospitalario de Medellín, reportan resistencia a nitrofurantoína en Proteus mirabilis, ampicilina en $P$. aeruginosa y $K$. pneumoniae; todas las resistencias reportadas en este trabajo fueron del $100 \%$ datos que son acordes a la literatura, si se tiene en cuenta que estos microorganismos poseen resistencia intrínseca a esos antibióticos por lo que no los afectaran.

En el estudio realizado por Mateus et al.(32), señalan que en las unidades de cuidados intensivos, en dos clínicas de Bucaramanga, encontraron en $P$. aeruginosa, altos porcentajes de resistencia frente a ampicilina/sulbactam $(93,3 \%)$, ceftriaxona $(82 \%)$ igualmente que en los estudios pasados evalúan antibióticos que no afectan dichos microorganismos dada su resistencia intrínseca.

Cabe destacar que los autores mencionan los reportes de resistencia bacteriana sobre dichos microorganismos que se realizan desde el laboratorio clínico, sin percatarse de la resistencia intrínseca que pueden tener y que por ello no debería hacerse dentro de los datos epidemiológicos de cada región e institución. La única salvedad encontrada en estos estudios fue la realizada por Vélez et al.(31), quienes indican que el uso de trimetoprima/sulfametoxazol frente a $P$. aeruginosa no aplicaba, dada la resistencia intrínseca de este microorganismo a este antibiótico, también es importante resaltar que muchos de estos trabajos se realizan con antibiogramas automatizados donde hay un panel general de antibióticos que no se puede cambiar, en estos casos a pesar que se analizan antibióticos a los cuales los microrganismos presentan resistencia intrínseca, no deberían ser reportados en el antibiograma o generar notas que aclaren este tipo de resistencia.

A nivel internacional también se logró observar reportes de antibióticos que no deberían usarse en determinados microorganismos dada su resistencia natural; ejemplo de ello son estudios donde se evalúo nitrofurantoína en $P$. mirabilis, ampicilina y cefuroxima en Enterobacter spp., también cefalosporinas en Enterococcus spp.(33), ampicilina en $K$. pneumoniae(34) o cefotaxime, ceftriaxona, trimetoprima/sulfametoxazol, amoxicilina en $P$. aeruginosa ${ }^{(35,36)}$. Aunque se considera que el manejo de los antibióticos a nivel clínico es algo que se ha establecido en 
centros hospitalarios, la literatura muestra que hay deficiencias en el manejo de los antibióticos y en el conocimiento en la administración de los mismos, Santander et al.(37), por ejemplo muestran que existe un desconocimiento en el manejo de los antimicrobianos en España evidenciando que en el $51,9 \%$ de casos, se realizaron tratamientos inadecuados, en otro $40,7 \%$ el tratamiento fue innecesario, y en un $35,2 \%$ la elección del antimicrobiano fue incorrecta, esto demostrando el desconocimiento en el uso de antibióticos. En el presente estudio logramos evidenciar un problema similar al observar que muchos informes de antibióticos no deberían realizarse. Chhorvoin(38) encontró que el $93 \%$ de médicos en Camboya tuvieron dificultades para seleccionar antibióticos apropiados para tratar infecciones comunes. El no conocer las resistencias intrínsecas de los microorganismos, lleva a que se reporten datos de sensibilidad a antibióticos sin tener presente que in vitro pueden ser activos, pero que clínicamente no son efectivos y presentarían falla terapéutica, tal y como sucede con E. faecalis que in vitro puede mostrar sensibilidad a cefalosporinas, pero clínicamente no es efectivo y por lo tanto no debería presentarse como susceptible(38).

Adicionalmente, es importante destacar que factores como: correcta elección, eficacia, seguridad, conveniencia, acceso, costos y tipo de infección, son indispensables en la toma de decisiones médicas, puesto que es el paciente quien finalmente puede verse afectado. Por ello, el uso del urocultivo y un correcto antibiograma es de gran importancia para evitar confusión en el personal de la salud y prevenir una inadecuada administración de tratamientos que conllevan a la presencia de infecciones persistentes o el favorecimiento de multiresistencia en microorganismos sensibles que hacen parte de la flora bacteriana ${ }^{(39)}$.

Aunque la debilidad del presente trabajo radica en que solo se tomaron datos de urocultivos de un único laboratorio, una revisión de la literatura científica evidenció un incorrecto tamizaje y uso de antibióticos frente a algunas especies de microorganismos; al igual que un incorrecto reporte de identificación de los urocultivos en los trabajos consultados, usando el género para mostrar porcentajes de resistencia, sin tener presente que un mismo género puede presentar especies con resistencias intrínsecas variables ${ }^{(8,11)}$.

En este estudio se pudo observar grandes diferencias en el uso de antibióticos evaluados, frente a lo descrito en el Manual de actualización en resistencia bacteriana, el cual se basa en la norma CLSI; encontrando que el $22,3 \%$ de los aislados evaluados tuvieron un procedimiento de identificación de susceptibilidad erróneo, dado que se consideraron antibióticos en bacterias naturalmente resistentes. Además, los antibióticos se evaluaron frente a todos los microorganismos mostrando así, que no hay claridad ni presencia de un panel de antibióticos que se empleen en forma selectiva frente a cada especie microbiana.

Teniendo en cuenta los datos aquí expuestos, la situación nacional e internacional, frente al uso de antibióticos, es importante resaltar que continuamente el personal de salud debería capacitarse y aclarar conceptos sobre las resistencias naturales en microorganismos, evitando así un reporte inadecuado de antibióticos en los momentos de tamizaje, que llevaría a un fallo en el tratamiento.

\section{Conclusión}

El estudio mostró que existe un problema en cuanto al manejo, reporte e interpretación de antibiogramas frente a microorganismos naturalmente resistentes, que podría favorecer el desarrollo de multirresistencia en microorganismos sensibles de la flora bacteriana. Una revisión de la bibliografía nacional e internacional, mostró reportes similares, ningún autor menciona resistencias intrínsecas, por esto, los datos de antibiótico resistencia serían sobreevaluados.

\section{Agradecimientos}

Los autores agradecen a la Corporación Universitaria Empresarial Alexander von 
Humboldt y al laboratorio clínico por el apoyo para la realización del presente trabajo.

\section{Conflicto de intereses}

Ninguno declarado por los autores.

\section{Referencias}

1. Acevedo-Barrios RL, Severiche-Sierra CA. Identificación de bacterias resistentes a di-bromo-mercurio aisladas de sedimentos en playas de Cartagena de Indias, caribe colombiano. Av Investig en Ing. 2013;10(2):73-9.

2. Organización Panamericana de la Salud. Vigilancia epidemiológica de las infecciones asociadas a la atención de la salud. 3 ed. Cabrera JE, Holder R, Ramón Pardo P, Stempliuk V, editors. Módulo III : información para gerentes y personal directivo. Washington: OPS; 2012.

3. Donado-Godoy P, Castellanos R, León M, Arevalo A, Clavijo V, Bernal J, et al. The Establishment of the Colombian Integrated Program for Antimicrobial Resistance Surveillance (COIPARS): A Pilot Project on Poultry Farms, Slaughterhouses and Retail Market. Zoonoses Public Health. 2015;62:58-69.

4. Nodarse R. Lectura interpretada del antibiograma Construed reading of the antibiogram. Rev Cuba Med Mil. 2013;42(3):502-6.

5. Moehring RW, Hazen KC, Hawkins MR, Drew RH, Sexton DJ, Anderson DJ. Challenges in preparation of cumulative antibiogram reports for community hospitals. J Clin Microbiol. 2015;53(9):2977-82.

6. Abera B, Kibret M, Mulu W. Knowledge and beliefs on antimicrobial resistance among physicians and nurses in hospitals in Amhara Region, Ethiopia. BMC Pharmacol Toxicol. 2014;15(1):26.

7. Leclercq R, Cantón R, Brown DF, Giske CG, Heisig P, MacGowan AP, et al. EUCAST Expert Rules in Antimicrobial Susceptibility Testing. Clin Microbiol Infect. 2011;19(2):1-34.

8. Murray JL, Kwon T, Marcotte EM, Whiteley M. Intrinsic antimicrobial resistance determinants in the superbug Pseudomonas aeruginosa. MBio. 2015;6(6):1-10.

9. Cercenado E. Enterococcus: resistencias fenotipicas y genotipicas y epidemiologicas en España. Enferm Infecc Microbiol Clin. 2011;29(Suppl. 5):59-65.

10. Perry JD, Butterworth LA, Nicholson A, Appleby MR, Orr KE. Evaluation of a new chromogenic medium, Uriselect 4, for the isolation and identification of urinary tract pathogens. J Clin Pathol. 2003;56(7):52831.

11. Clinical and Laboratory Standards Institute. M100-S23 Performance Standards for Antimicrobial. Pennsylvania: Wayne; 2015. 1-238 p.

12. Muñoz-Delgado Á, Valderrama-Duarte C. Manual de procedimientos para la determinación de susceptibilidad antibiótica en patógenos de importancia hospitalaria. Bogotá: Instituto Nacional de Salud; 2012. 1-30 p.
13. Frieri M, Kumar K, Boutin A. Antibiotic resistance. J Infect Public Health. 2017;10(4):369-78.

14. Dodds DR. Antibiotic resistance: A current epilogue. Biochem Pharmacol. 2017;134(December):139-46.

15. Orrego C, Henao C, Cardona J. Prevalencia de infección urinaria, uropatógenos y perfil de susceptibilidad antimicrobiana. Acta Médica Colomb [Internet]. 2014;39(4):352-8.

16. Alvarez-Barranci LC. Infecciones de vías urinarias en el Hospital Universidad del Norte. Salud Uninorte. 2007;23(1):9-18.

17. Okragla E, Szychowska K, Wolska L. [Mechanisms of urinary tract sterility maintenance]. Postep Hig Med Dosw. 2014;68(0):684-94.

18. Alós JI. Epidemiología y etiología de la infección urinaria comunitaria. Sensibilidad antimicrobiana a determinados patógenos y significado clínico de la resistencia. Medes. 2005;23(4):3-8.

19. Vélez-Echeverri C, Serna-Higuita LM, Serrano AK, Ochoa-García C, Rojas-Rosas L, Bedoya AM, et al. Resistance profile for pathogens causing urinary tract infection in a pediatric population, and antibiotic treatment response at a University Hospital, 20102011. Colombia Médica. 2014;45(1):39-44.

20. González A, Dávila R, Acevedo O, Ramírez ME, Gilbaja S, Valencia $\mathrm{C}$, et al. Infección de las vías urinarias: prevalencia, sensibilidad antimicrobiana y factores de riesgo asociados en pacientes con diabetes mellitus tipo 2. Rev Cuba Endocrinol. 2014;25(2):57-65.

21. Blanco VM, Maya JJ, Correa A, Perenguez M, Muñoz JS, Motoa $G$, et al. Prevalencia y factores de riesgo para infecciones del tracto urinario de inicio en la comunidad causadas por Escherichia coli productor de betalactamasas de espectro extendido en Colombia. Enferm Infecc Microbiol Clin. 2016;34(9):559-65.

22. Avilés C, Betancour P, Velasco CL, Godoy R, Barthel E, Martínez F. Factores asociados a infecciones urinarias producidas por enterobacterias productoras de \&amp;\#946;-lactamasas de espectro extendido: una cohorte prospectiva. Rev Chil infectología. 2016;33(6):628-34.

23. Hernández-Marco R, Guillén-Olmos E, Bretón-Martínez JR, Giner-Pérez L, Casado-Sánchez B, Fujkova J, et al. Infección urinaria febril adquirida en la comunidad por bacterias productoras de betalactamasas de espectro extendido en niños hospitalizados. Enferm Infecc Microbiol Clin. 2017;35(5):287-92.

24. Chacón PM, Viguria FB, Aniceto ÁA, Cervera C, Méndez $\mathrm{C}$, Rodriguez $\mathrm{C}$, et al. Infección del tracto urinario en receptores de trasplante renal Urinary tract infection in renal transplant recipients. An Fac med. 2017;78(1):11-6.

25. Marlene J, Camulombo C, Alvarez BR, Jala MC, Amarilys G, Chantez R, et al. Evaluación de la resistencia antimicrobiana de cepas de Escherichia coli causantes de infecciones urinarias en la provincia de Huambo, Angola. Rev Cuba Ciencias Biológicas. 2015;4(2):71-7.

26. Blanco VM, Maya JJ, Correa A, Perenguez M, Motoa G, Pallares CJ, et al. HHS Public Access. 2016;34(9):55965. 
27. Machado J, Murillo M. Evaluación de sensibilidad antibiótica en urocultivos de pacientes en primer nivel de atención en salud de Pereira. Rev Salud Pública. 2012;14(4):710-9.

28. Navarro F, Miró E, Mirelis B. Lectura interpretada del antibiograma de bacilos gramnegativos no fermentadores. Enferm Infecc Microbiol Clin. 2010;28(10):638-45.

29. Leal AL, Cortés JA, Arias G, Ovalle MV, Saavedra SY, Buitrago G, et al. Emergencia de fenotipos resistentes a cefalosporinas de tercera generación en Enterobacteriaceae causantes de infección del tracto urinario de inicio comunitario en hospitales de Colombia. Enferm Infecc Microbiol Clin. 2013;31(5):298-303.

30. Saavedra SY, Carolina D, González MN, Realpe ME. Caracterización de aislamientos de Pseudomonas aeruginosa productores de carbapenemasas aislados en siete departamentos de Colombia. Biomédica. 2014;34(Supl. 1):1-25.

31. Vélez-Echeverri C, Serna-Higuita LM, Serrano AK, Ochoa-García C, Rojas-Rosas L, Bedoya AM, et al. Resistance profile for pathogens causing urinary tract infection in a pediatric population, and antibiotic treatment response at a University Hospital, 20102011. Colomb Med. 2014;45(1):39-44.

32. Mateus J, Francisco L, Gladys G, Narvaez E. Resistencia a los antibioticos en dos unidades de cuidados intensivos de Bucaramanga (Colombia). Salud Soc Uptc. 2014;1(1):12-8.

33. Catal F, Bavbek N, Bayrak 0, Karabel M, Karabel D, Odemis E, et al. Antimicrobial resistance patterns of urinary tract pathogens and rationale for empirical therapy in Turkish children for the years 2000-2006. Int Urol Nephrol. 2009;41(4):953-7.

34. Yuksel S, Ozturk B, Kavaz A, Ozcakar ZB, Acar B, Guriz $\mathrm{H}$, et al. Antibiotic resistance of urinary tract pathogens and evaluation of empirical treatment in Turkish children with urinary tract infections. Int J Antimicrob Agents. 2006;28(5):413-6.

35. Akram M, Shahid M, Khan A. Etiology and antibiotic resistance patterns of community-acquired urinary tract infections in JNMC Hospital Aligarh, India. Ann Clin Microbiol Antimicrob. 2007;6:4.

36. Bogantes R, Rodríguez J. Resistencia bacteriana a los antibióticos en infecciones del tracto urinario bajo, en pacientes de consulta externa en el área de salud Palmares. Fármacos. 2004;17:1-2.

37. Croche-Santander B, Campos-Alonso E, SánchezCarrión A, Marcos-Fuentes L, Díaz-Flores I, Vargas JC, et al. Adecuación de la prescripción de antimicrobianos en población pediátrica en un servicio de urgencias hospitalario. An Pediatría. 2017; In Press:1-7.

38. Chhorvoin, Vlieghe E, McLaughlin JC, Daily F, McLaws ML. Antibiotic prescribing practices: A national survey of Cambodian physicians. Am J Infect Control [Internet]. 2016;44(10):1144-8. Available from: http://dx.doi.org/10.1016/j.ajic.2016.03.062

39. Souza RB, Trevisol DJ, Schuelter-Trevisol F. Bacterial sensitivity to fosfomycin in pregnant women with urinary infection. Brazilian J Infect Dis. 2015;19(3):319-23. 